\title{
Towards Self-Verification in Finite Difference Code Generation
}

\author{
Jan Hückelheim \\ Imperial College London \\ London, UK \\ j.hueckelheim@imperial.ac.uk \\ Navjot Kukreja \\ Imperial College London \\ London, UK \\ n.kukreja@imperial.ac.uk \\ Stephen Siegel \\ University of Delaware \\ Newark, DE, USA \\ siegel@udel.edu
}

\author{
Ziqing Luo \\ University of Delaware \\ Newark, DE, USA \\ ziqing@udel.edu \\ Michael Lange \\ Imperial College London \\ London, UK \\ michael.lange@imperial.ac.uk \\ Matthew Dwyer \\ University of Nebraska \\ Lincoln, NE, USA \\ matthewbdwyer@unl.edu
}

\author{
Fabio Luporini \\ Imperial College London \\ London, UK \\ f.luporini12@imperial.ac.uk \\ Gerard Gorman \\ Imperial College London \\ London, UK \\ g.gorman@imperial.ac.uk \\ Paul Hovland \\ Argonne National Laboratory \\ Lemont, IL, USA \\ hovland@mcs.anl.gov
}

\begin{abstract}
Code generation from domain-specific languages is becoming increasingly popular as a method to obtain optimised lowlevel code that performs well on a given platform and for a given problem instance. Ensuring the correctness of generated codes is crucial. At the same time, testing or manual inspection of the code is problematic, as the generated code can be complex and hard to read. Moreover, the generated code may change depending on the problem type, domain size, or target platform, making conventional code review or testing methods impractical. As a solution, we propose the integration of formal verification tools into the code generation process. We present a case study in which the CIVL verification tool is combined with the Devito finite difference framework that generates optimised stencil code for PDE solvers from symbolic equations. We show a selection of properties of the generated code that can be automatically specified and verified during the code generation process. Our approach allowed us to detect a previously unknown bug in the Devito code generation tool.
\end{abstract}

\section{CCS CONCEPTS}

- Software and its engineering $\rightarrow$ Formal methods; Software verification; Automated static analysis; Concurrent programming structures; Source code generation;

Permission to make digital or hard copies of all or part of this work for personal or classroom use is granted without fee provided that copies are not made or distributed for profit or commercial advantage and that copies bear this notice and the full citation on the first page. Copyrights for components of this work owned by others than the author(s) must be honored. Abstracting with credit is permitted. To copy otherwise, or republish, to post on servers or to redistribute to lists, requires prior specific permission and/or a fee. Request permissions from permissions@acm.org.

Correctness'17, November 12-17, 2017, Denver, CO, USA

(C) 2017 Copyright held by the owner/author(s). Publication rights licensed to Association for Computing Machinery.

ACM ISBN 978-1-4503-5127-0/17/11 . .\$15.00

https://doi.org/10.1145/3145344.3145488

\section{KEYWORDS}

HPC, Code generation, Verification, Specification, Formal methods, Symbolic execution, Equivalence checking

\section{ACM Reference Format:}

Jan Hückelheim, Ziqing Luo, Fabio Luporini, Navjot Kukreja, Michael Lange, Gerard Gorman, Stephen Siegel, Matthew Dwyer, and Paul Hovland. 2017. Towards Self-Verification in Finite Difference Code Generation. In Proceedings of Correctness'17: First International Workshop on Software Correctness for HPC Applications (Correctness'17). ACM, New York, NY, USA, 8 pages. https://doi.org/10.1145/3145344.3145488

\section{INTRODUCTION}

Automated code generation is a technology that can combine the productivity of high-level programming languages with the performance of optimised, platform-specific low-level code [20]. In combination with domain-specific languages it is increasingly used to enable domain experts to rapidly develop new scientific modelling applications that scale well on high-performance computing (HPC) resources and emerging architectures [3, 17, 24, 35]. It is therefore not surprising that several Gordon Bell Prize finalist submissions in 2016 used code generation techniques to achieve cutting-edge performance results $[30,41]$.

In a workflow that uses code generation techniques, the responsibility for implementing low-level performance optimisation techniques is shifted from the domain experts to the developers of code generation tools. At the same time, the responsibility for ensuring the correctness of said optimisations should also be removed from the user. If successful, such a shift could be an important advantage of code generation tools over manually implemented application code, as the complexity of realistic HPC applications is becoming increasingly hard to establish [15].

Conventional manual approaches such as testing and code inspections are already faced with increasing challenges, and are especially troublesome for generated code. The automatic application of common performance optimisation strategies, such as loop blocking, might result in vastly more complex 
low-level code that splits the computation into a number of loops with complex derived loop bounds. Moreover, runtime code generation allows various optimisations to be applied depending on external factors, such as user inputs, the computing environment, and the complexity of the computation. Any test or code review must therefore be repeated for a large parameter space. Instead, we propose to combine formal verification methods with modern code generation tools to facilitate the automatic verification of crucial properties during code generation.

As a first step towards this goal, we investigate ways to apply the verification tool CIVL [40] to high-performance stencil kernels generated by the symbolic finite difference (FD) framework Devito [29]. The Devito framework generates low-level $\mathrm{C}$ code from high-level symbolic problem definitions in Python, while applying a range of automated code transformations to increase the performance of the generated kernels. We manually instrumented the generated $\mathrm{C}$ code with specifications and assertions, allowing us to verify the absence of data races, deadlocks, segmentation faults and undefined behaviour.

While this is not enough to establish all aspects of correctness of the generated code, it allowed us to verify that the optimised and parallelised code produced by Devito is equivalent to a human-readable, platform-independent baseline implementation whose correctness can be more readily checked by a domain expert. We also detected a previously unknown bug in Devito that caused the generation of code with faulty loop blocking for certain combinations of domain and cache block sizes.

\section{CIVL VERIFICATION TOOL}

The Concurrent Intermediate Verification Language (CIVL) [40] is a verification framework for C programs, including parallel C programs that use MPI [27], OpenMP [32], POSIX threads (Pthreads) [1], and CUDA [31]. The CIVL framework includes the intermediate verification language CIVL-C, a model checker to verify programs written in CIVL-C, and a front-end to translate $\mathrm{C}$ programs into the CIVL-C language.

The CIVL-C programming language is based on sequential $\mathrm{C}$, and adds primitives to specify concurrency, assertions, assumptions, and other constructs for specification and verification. While most sequential $\mathrm{C}$ programs are also legal CIVL-C programs, the concurrency model in CIVL-C differs from that in C. In CIVL-C, a function can be defined in any scope and can be spawned as a process. This flexible mechanism is used to model OpenMP, MPI, Pthreads and CUDA, and programs that combine more than one of those.

CIVL-C provides primitives such as \$assume and \$assert to specify the intended behavior of programs. The \$assume primitive instructs the verifier to ignore executions in which the assumption does not hold. Assumptions are expressed in a first-order logic and may use the \$forall and \$exists quantifiers. The type qualifiers \$input and \$output are used to declare input and output variables. The \$assert primitive extends the assert function of $\mathrm{C}$. If an assertion can fail for any program input under the given assumptions, then CIVL will report this as an assertion violation. In addition, the specification of a program can be expressed by providing a second program which is expected to be functionally equivalent to the first, i.e., the two programs should produce the same output when given the same (arbitrary) input.

The model checker in CIVL uses symbolic execution to check safety properties and functional equivalence of CIVL-C programs. Examples of safety properties include assertion violations, improper pointer dereferencing or out-of-bound array access, division by zero, and properties such as dataraces in OpenMP programs. To verify functional equivalence, the two programs are incorporated as procedures into a single program. The two procedures share the same inputs, but write to different output variables. The procedures are invoked sequentially and the outputs are asserted to be equal. The model checker is applied to this combined program to check that the assertions hold for all possible inputs. The CIVL framework automates this entire process.

The model checker attempts to explore all reachable states of a CIVL-C program. An error will be reported as soon as the model checker reaches a state where a property violation occurs. A common challenge in model checking is the exponential growth (with the number of variables, processes, or other program components) in the number of reachable states. CIVL uses Partial Order Reduction (POR) [14] techniques to mitigate this state explosion problem. These techniques reduce the number of states that need to be explored by choosing only one transition sequence from a set of commutative sequences that must result in the same state. In addition, a "simplifier" is used for OpenMP parallel loops to perform a loop dependence analysis, which often permits CIVL to check the safety properties statically and replace the parallel loop with an equivalent sequential loop.

Nevertheless, like many other model checkers, CIVL requires relatively small concrete bounds on the number of processes and input sizes, and relies on the Small Scope Hypothesis [18], which states that program defects usually manifest themselves in program executions with small configurations.

\section{DEVITO}

Devito [21, 29] is an open-source domain-specific framework for solving partial differential equations (PDE) in symbolic form using the finite difference method. Devito generates efficient stencil code from high-level symbolic problem definitions, giving users the freedom to easily change the numerical discretisation of a given operator, while automating wellknown performance optimisation techniques. Despite being originally developed for seismic imaging workflows, Devito is capable of expressing a range of scientific problems due to an extensible high-level API that utilises symbolic Python (SymPy) [28].

Devito provides a set of custom Python classes to allow users to symbolically define complex stencil operators through a set of symbolic expressions. These expressions are passed to 
an Operator object that incrementally lowers the symbolic definitions to semantically equivalent $\mathrm{C}$ code and utilises Just-in-Time (JIT) compilation to perform the resulting computation from within the Python interpreter. During the code generation process, Devito applies several performance optimisations to the intermediate representation of the code through two internal "engines": The Devito Symbolic Engine (DSE) aims to reduce the overall operation count of the generated kernel, while the Devito Loop Engine (DLE) performs well-known loop-level optimisation techniques.

Even in the most basic setup, the DSE applies factorisation to collect common finite difference weights and thus reduces the overall operation count. Moreover, problem-specific constants are hard-coded and partial evaluation is used to perform as much work as possible at compile-time. In more advanced settings, the DSE applies an ordered sequence of compiler passes, including common sub-expression elimination, hoisting of time-invariant sub-expressions [2, 26], and detection of cross-iteration redundancies [12].

The DLE primarily adds shared-memory parallelism and SIMD vectorisation to the generated loops by using OpenMP pragmas. Depending on the underlying architecture, different pragmas may be generated (e.g., on Intel KNL platforms, spatial loops are collapsed to maximise the parallel workload), while SIMD vectorisation is applied to the innermost loop.

The DLE is also responsible for cache and memory bandwidth optimisations. The most important transformation provided by the DLE is "loop blocking", a standard technique to reduce the number of cache misses [22]. The DLE currently supports blocking of spatial loops, while support for "time tiling" (i.e., blocking across both the time and space loops) is planned for future versions. An important implementation detail is the management of remainder iterations. If the loop trip count is not a multiple of the chosen block size, then one or more remainder iterations need to be executed. This is often implemented by generating "remainder loop nests". Finally, the DLE pulls the loop bodies that are identical between all loops into a separate routine. The DLE optimisations in Devito have been shown to yield significant speedups compared to the non-optimised code, e.g., a speedup of $2.7 \times$ in a seismic imaging application [29].

\section{TEST CASE}

We apply CIVL to a set of $\mathrm{C}$ codes that are generated by Devito for solving the convection-diffusion equation for incompressible flows with constant diffusion coefficient $d$ and a uniform constant velocity $a$ given as

$$
\frac{\partial u}{\partial t}=d\left(\frac{\partial^{2} u}{\partial x^{2}}+\frac{\partial^{2} u}{\partial y^{2}}\right)-a\left(\frac{\partial u}{\partial x}+\frac{\partial u}{\partial y}\right) .
$$

In the finite difference method, the first spatial derivative in Equation (1) is replaced by a quotient that is a good approximation for the derivative if the step size $h$ is small. In this test case, we use the second-order backward quotient

$$
\frac{\partial u}{\partial x} \approx \frac{3 u(t, x, y)-4 u(t, x-h, y)+u(t, x-2 h, y)}{2 h} .
$$

The second derivative is approximated by the central difference quotient

$$
\frac{\partial^{2} u}{\partial x^{2}} \approx \frac{u(t, x+h, y)+u(t, x-h, y)-2 u(t, x, y)}{h^{2}} .
$$

We use a first-order backward difference quotient for the temporal derivative to obtain an explicit procedure to compute an approximate solution to the PDE. The kernels discussed below implement this in the following variants:

- Classic: a textbook implementation of a convectiondiffusion finite difference solver, implemented in Listing 1 . The convection and diffusion coefficients, time step size, and grid resolution are defined in the beginning of the code. The computation of each time step happens in a two-step process: First, unnew is computed based on the values in u_old. Second, the new values are copied into u_old.

- Basic: Devito-generated code with DLE and DSE switched to basic mode. As described in $\S 3$, even the basic mode groups common terms and precomputes coefficients through partial execution. The two-step updating process is replaced by one where the source and target arrays swap their roles without data copies. The result is shown in Listing 3. The complete script that is used to call Devito and generate this example code is shown in Listing 2.

- Parallel: Devito-generated code with DLE and DSE set to its advanced mode, shown in Listing 4. This includes shared-memory parallelisation using OpenMP. Due to a limitation in the current version of CIVL, OpenMP SIMD pragmas needed to be removed for successful verification, as this is not yet supported.

- Advanced: As an intermediate step, we verify the equivalence of the basic code and the code described in parallel, but with all OpenMP pragmas removed.

The main loops performing the interior computation are discussed below. Other parts of the code such as boundary or error handling are left away for readability.

\section{METHOD}

Following our proposed method, the verification is split into two stages. First, the correctness of the baseline implementation is established using traditional methods as discussed in $§ 5.1$. Second, the equivalence of generated code with the baseline code is shown using formal verification as shown in $\S 5.2$.

\subsection{Correctness of Baseline Code}

The baseline code is manually written, following a conventional finite difference approach. Since this code is never used to perform large-scale computations, the developer can focus entirely on readability and clarity. The code does not need to adapt to different computing platforms, and therefore does not need to change often. As a result, conventional testing and a manual code review can be sufficient to gain confidence in the correctness of this code. 
Listing 1: Hand-written code

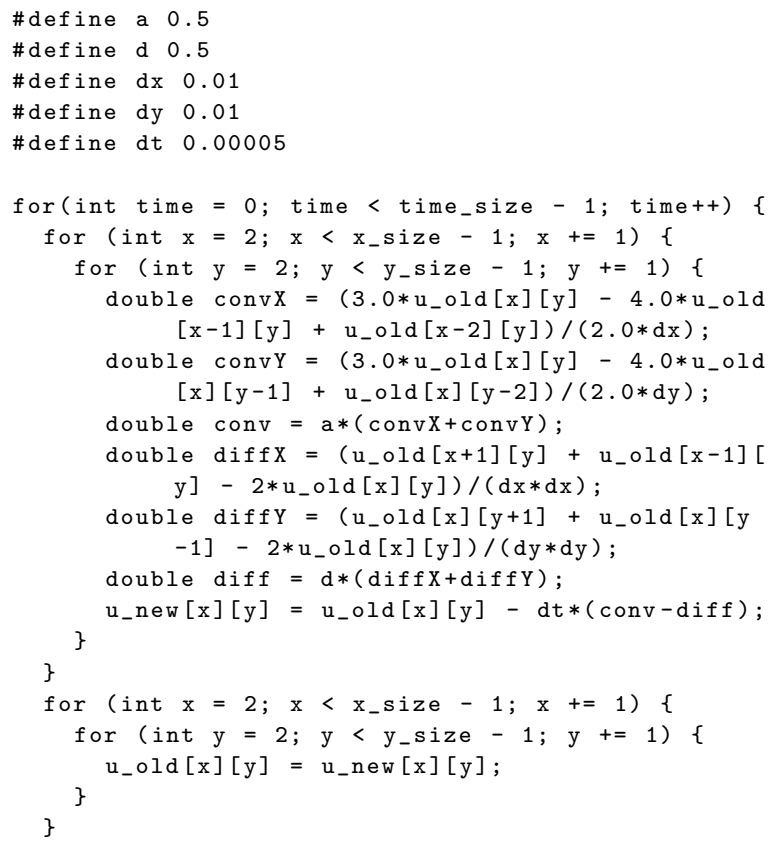

\section{Listing 2: Devito DSL}

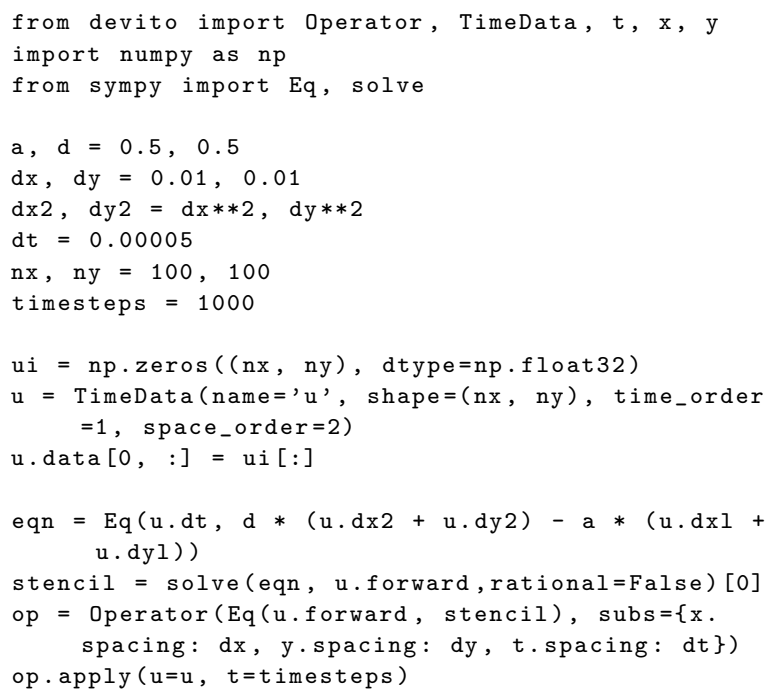

In some cases, it may be appropriate to use the method of manufactured solutions [37], or even a human-assisted verification approach [8]. Still, this effort needs to be undertaken only for the relatively simple baseline code.

\subsection{Correctness of Generated Code}

The $\mathrm{C}$ functions generated by Devito are normally called from within Python. To verify properties of these functions,
Listing 3: Code for DLE/DSE=basic

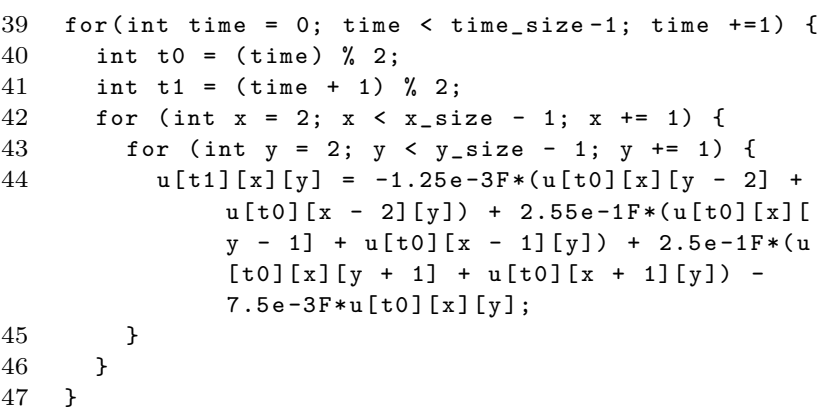

we use a driver program, which contains a main function that calls the generated $\mathrm{C}$ functions. This driver program is shown in Listing 5.

We use CIVL to check the functional equivalence of the baseline code and the generated code variants for arbitrary inputs with a bounded number of threads and bounded domain and block sizes. This is achieved by instrumenting the driver program with CIVL-C primitives \$input to declare input variables, \$output to specify program outputs, and \$assume to provide bounds for some inputs. The primitive \$elaborate (expr) instructs the model checker to explore all possible concrete values of expr at the specific location. In addition, CIVL checks the safety properties as described in $\S 2$ for the baseline and all generated programs.

For the OpenMP-parallel variant of the generated code, the simplifier in CIVL is able to prove that in any two distinct iterations of these loops, the set of memory locations written in one iteration is disjoint from the set of memory locations read and written in the other iteration. This results in a successful sequentialisation of the loops, and thus a verification that is valid for arbitrary numbers of threads.

The time and memory that is necessary for the verification depends on the domain size bounds, as shown in $\S 6.2$. The verification results are presented in $\S 6$.

\subsection{Remaining Limitations}

There are some steps that currently have to be performed manually on a Devito-generated code to enable verification with CIVL:

- Devito uses an external profiling library, calls to which have been removed manually.

- In some cases, hard-coded problem-specific array indices are used to implement boundary conditions, which had to be manually encoded as a function of the problem size to enable the verification for arbitrary problem sizes up to some bound.

- Devito passes a one-dimensional data array from Python to $\mathrm{C}$, and a pointer to the start of that array is cast into a three-dimensional array pointer. CIVL does not currently support such a cast operation, and this operation was hidden from CIVL using preprocessor pragmas. 
Listing 4: Code for DLE/DSE=advanced

\section{(a) Blocked loop}

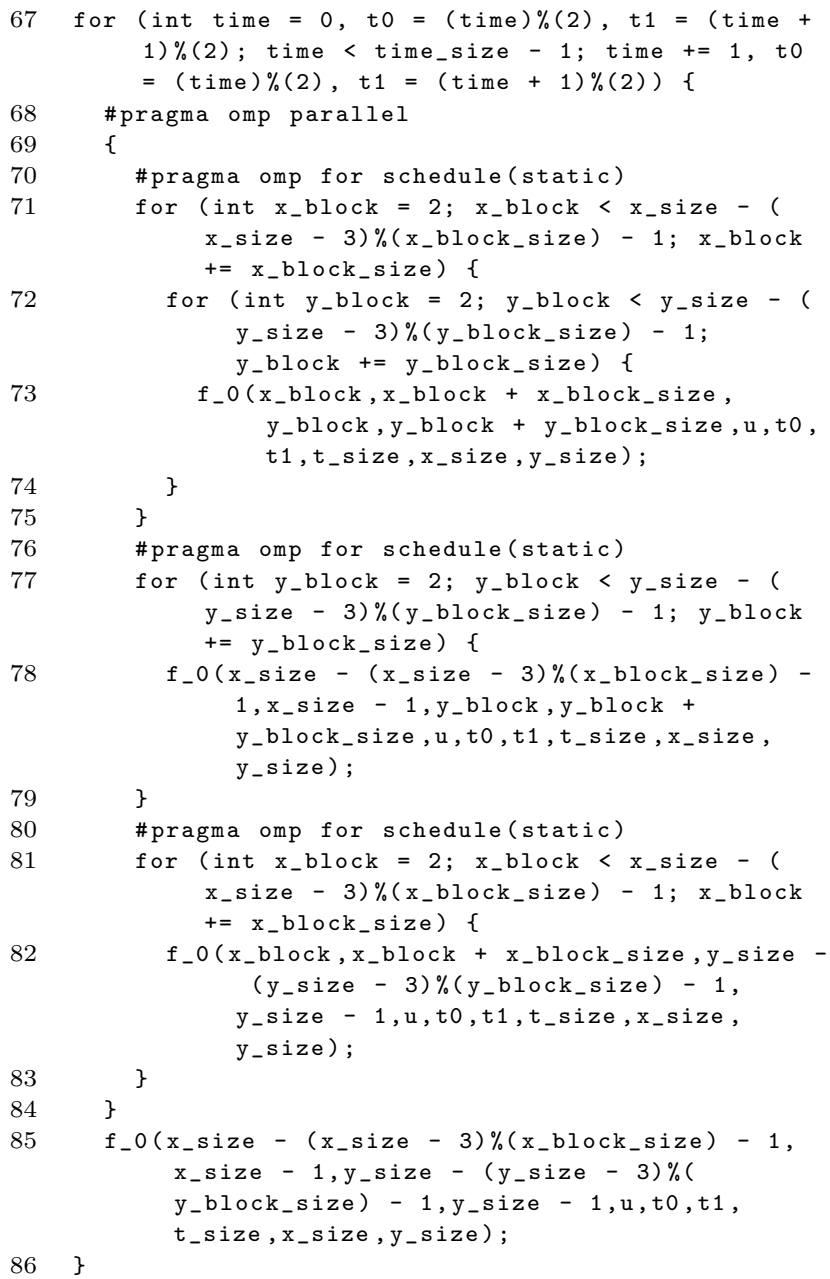

\section{(b) Outlined function}

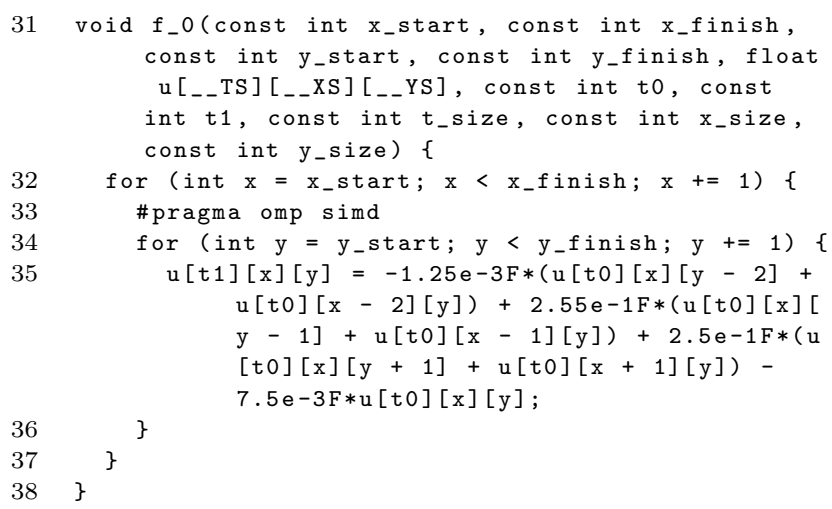

\section{Listing 5: Driver program}

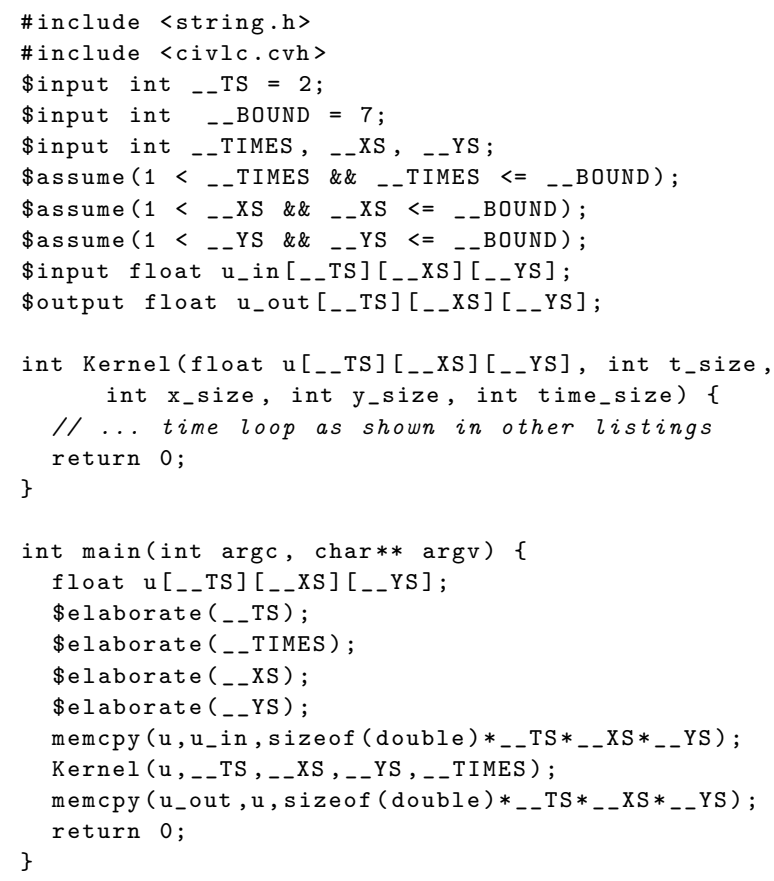

- The Devito-generated code contains commands that control the rounding of floating point numbers (e.g. FLUSH_TO_ZERO), but CIVL does not currently support codes that contains such statements, and furthermore does not model roundoff errors and instead assumes real arithmetic.

- Finally, we removed SIMD and restrict keywords, as CIVL lacks support for these currently.

We expect that the above manual modifications can be avoided in future versions of Devito and CIVL. In addition, there are some more fundamental limitations: We assume that neither the $\mathrm{C}$ compiler nor other parts of the environment introduce new bugs, we do not model or test for roundoff errors, and we only show correctness for problem sizes up to some limit.

\section{RESULTS}

We detected a previously unknown bug in Devito's loop blocking algorithm, shown in $\S 6.1$. After fixing this bug, the verification succeeded as detailed in $\S 6.2$.

\subsection{Revealed Bug in Generated Code}

The loop blocking algorithm in Devito contained a bug that could result in out-of-bounds array access. For the test case presented in $\S 4$, this happened if the domain size was exactly 2 above a number that is divisible by the block size. Formally, for a domain of size $n_{x} \times n_{y}$ with block sizes $b_{x}$ and $b_{y}$, the bug occurred if

$$
\left(n_{x}-2\right) \% b_{x}=0 \vee\left(n_{y}-2\right) \% b_{y}=0 .
$$



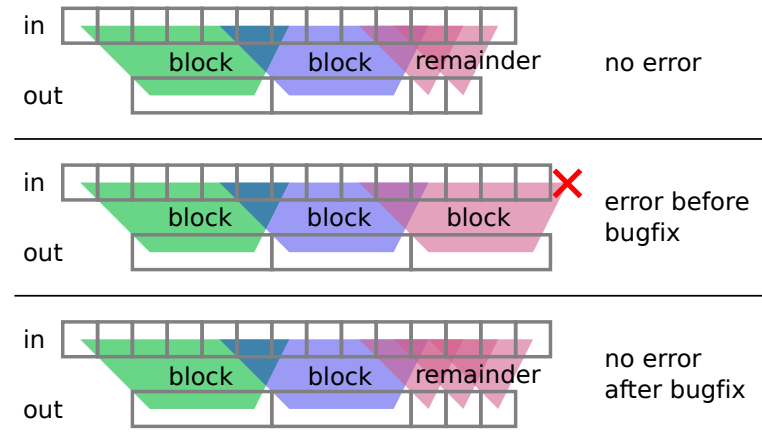

Figure 1: Illustration of the memory access pattern of the stencil codes. Each block reads from a range of indices (above) that is by three items larger than the range of indices that it writes to (below). An error occurred whenever the last block would write exactly up to the end of the domain, and read out-of-bounds. After fixing the bug, the last block is correctly replaced by a remainder loop.

The cause of this bug lies in the generation of remainder loops. In our test case, each time step requires a nested loop over the $x$ and $y$ dimensions. The number of iterations in the $x$ and $y$ dimensions is given by $n_{x}-3$ and $n_{y}-3$, respectively. The offset of 3 stems from the fact that the backward difference quotient shown in (2) requires an offset of two cells at the top left boundary, while the central difference quotient in (3) requires an offset of one cell on every side. Consequently, a total of three rows and columns form the boundary of the domain and have to be computed separately.

The remaining $n_{x}-3$ or $n_{y}-3$ iterations must be split into blocks of the given block size. If this number can not be evenly divided by the block size, a remainder loop has to be used. The loop bounds, however, were incorrectly based on an iteration count of $n_{x}-2$ and $n_{y}-2$. This was caused by an omission inside the code generator, which resulted in only the offsets of the backward difference quotient being subtracted from the domain size. As a consequence, the generated code would attempt to read beyond the right and bottom of the domain as illustrated in Figure 1.

For realistic block sizes, the bug often isn't triggered. For example, for a block size of 64 , a mesh with randomly chosen size has a $98.4375 \%$ chance of not triggering the bug. For meshes where the bug is triggered, the out-of-bounds access may result in a solution field that is not obviously wrong, since the whole domain is stored in a contiguous chunk of memory, and even an out-of-bounds read access will thus often return a value that is not obviously wrong. CIVL detected this out-of-bounds access as a violation of the safety properties.

\subsection{Verification Results after Bugfix}

After discovering this bug, Devito was updated to produce code with correctly applied loop blocking. CIVL can now verify for arbitrary inputs up to a maximum domain size of 8 by 8 that the machine-generated OpenMP program is functionally equivalent to the hand-written program, and

\begin{tabular}{|c|c|c|c|}
\hline Matrix sizes & Time (sec) & States & Prover \\
\hline $2<=\ldots$ TS,_-_XS, _-_YS $<=5$ & 11.14 & 316929 & 27 \\
\hline $2<={ }_{--} \mathrm{TS}_{\text {-_- }} \mathrm{XS},{ }_{--} \mathrm{YS}<=6$ & 46.56 & 742423 & 33 \\
\hline $2<=\_$TS,__XS, _-_YS $<=7$ & 194.57 & 1568961 & 39 \\
\hline $2<={ }_{--} \mathrm{TS}_{\text {-__ }} \mathrm{XS},{ }_{--} \mathrm{YS}<=8$ & 707.38 & 2976384 & 45 \\
\hline
\end{tabular}

Figure 2: Results of using CIVL to check functional equivalence between the hand written and advanced Devito code. Domain size bounds are shown in "Matrix sizes". The number of states explored by the CIVL model checker is shown in "States". To reason about complex symbolic expressions during the verification, CIVL invokes external provers (the number of invocations is shown in "Prover").

that all safety properties listed in $\S 2$ hold for both programs. Performance data of the verification is presented in Figure 2.

The verification is sound for any number of OpenMP threads executing the parallel regions, as the OpenMP simplifier is able to sequentialise all parallel loops in this test case. This is done by establishing that for any two iterations $i$ and $j(i \neq j)$, all objects being read in $i$ are elements of array $u[t 0]$ while the only object being written in $j$ is an element of array $u[t 1]$. These two are different arrays because $t 0=($ time $) \%(2), t 1=($ time +1$) \%(2))$ entails $t 0 \neq t 1$. Therefore, it is impossible that different iterations of the loop can read and write the same object simultaneously.

\section{RELATED WORK}

Previous work [8] demonstrated a one-dimensional wave equation solver that implements a provably correct discretisation of the wave equation, with verified bounds for convergence and roundoff error. The approach required a considerable amount of problem-specific manual reasoning, and the application of this approach to a code generation environment would be challenging.

Instead, we rely on a trusted implementation that is simple enough to be exhaustively tested by a domain expert, and verify the functional equivalence of the generated optimised codes with that baseline. This approach could also be used for other code generation frameworks such as FEniCS [25] and Firedrake [36].

A popular performance optimisation technique for stencil computations is polyhedral compilation. The automated verification of such transformations was previously demonstrated in [38] by proving the equivalence of the original and transformed codes. Other authors successfully attempted the verification of GPU parallelisation [6] and verified lifting to domain-specific languages [19] of stencil computations.

The CIVL-C language that was mentioned in $\S 2$ was developed to facilitate the verification of programs written in different programming languages and parallelisation dialects within a shared verification framework. Other intermediate verification languages have been developed, such as Boogie [5], used by the SMACK [34] verifier to verify LLVM IR programs, or the WhyML language, used by the Why3 platform [7] to verify C, Java, and Ada programs, and used 
by the Frama-C [11] platform to verify sequential C programs. Boogie and WhyML are typically used for deductive verification. In contrast, CIVL-C was developed to support model checking and symbolic execution, which are more appropriate techniques for the verification of concurrent programs.

Unlike CIVL, many model checking and symbolic execution tools are developed for one specific concurrency model. This applies to model checkers including ISP [16] (C with MPI), Java PathFinder [33] (Java), and DiVinE [4] (C with Pthreads), as well as tools based on concolic testing [39] including CONCREST [13], which checks multi-threaded C programs, and GKLEE [23], which extends KLEE [10] to check CUDA/C $/ \mathrm{C}++$ programs.

In [9], the authors describe a method to check the parallelisation of a program through a nondeterministic sequential specification (NDSeq), where the user-provided nondeterminism models the parallel behaviour. In contrast, CIVL only requires a sequential specification to check the correctness of a parallel implementation.

\section{CONCLUSIONS}

We demonstrate in this work that combining automatic verification and code generation can increase the reliability of generated code by detecting bugs that are hard to find with conventional testing or manual code inspection.

In the future, we plan to fully automate the verification of generated codes (possibly triggered by a debug command line flag in the code generation tool), and to increase the number of properties that can be automatically verified. Other properties that may be possible to verify are whether a numerical scheme is total-variation-diminishing (a property that guarantees stability), whether some code implements the adjoint of some other code, whether a compact higher order stencil is equivalent to a conventional higher order stencil, or whether a numerical discretisation is accurate for all polynomials of some order.

It may also be interesting to verify the equivalence of the codes under the assumption of floating point arithmetic, or failing that, to establish bounds for the roundoff errors in the different implementations.

\section{ACKNOWLEDGMENTS}

This work was supported by the Imperial College London Intel Parallel Computing Centre, the Engineering and Physical Sciences Research Council (EPSRC) under grant number EP/L000407/1, by the U.S. Department of Energy, Office of Science, Office of Advanced Scientific Computing Research, Applied Mathematics and Computer Science programs under contract number DE-AC02-06CH11357 and contract number DE-SC0012566, and by U.S. National Science Foundation Award CCF-1319571.

\section{REFERENCES}

[1] IEEE POSIX 1003.1c standard. 1995. POSIX Threads. http: //www.unix.org/version3/ieee_std.html. (1995). Accessed Oct. $30,2015$.
[2] Alfred V. Aho, Ravi Sethi, and Jeffrey D. Ullman. 1986. Compilers, Principles, Techniques, and Tools. Addison-Wesley.

[3] A Arbona, B Miñano, A Rigo, C Bona, C Palenzuela, A Artigues, C Bona-Casas, and J Massó. 2017. Simflowny 2: An upgraded platform for scientific modeling and simulation. arXiv preprint arXiv:1702.04715 (2017).

[4] Jiri Barnat, Lubos Brim, Vojtech Havel, Jan Havlícek, Jan Kriho, Milan Lenco, Petr Rockai, Vladimír Still, and Jirí Weiser. 2013. DiVinE 3.0 - An Explicit-State Model Checker for Multithreaded C \& C ++ Programs. In Computer Aided Verification - 25th International Conference, CAV 2013, Saint Petersburg, Russia, July 13-19, 2013. Proceedings (Lecture Notes in Computer Science), Natasha Sharygina and Helmut Veith (Eds.), Vol. 8044. Springer, 863-868.

[5] Michael Barnett, Bor-Yuh Evan Chang, Robert DeLine, Bart Jacobs, and K. Rustan M. Leino. 2005. Boogie: A Modular Reusable Verifier for Object-Oriented Programs. In Formal Methods for Components and Objects, 4th International Symposium, FMCO 2005, Amsterdam, The Netherlands, November 1-4, 2005, Revised Lectures (Lecture Notes in Computer Science), Frank S. de Boer, Marcello M. Bonsangue, Susanne Graf, and Willem P. de Roever (Eds.), Vol. 4111. Springer, 364-387.

[6] Adam Betts, Nathan Chong, Alastair F. Donaldson, Jeroen Ketema, Shaz Qadeer, Paul Thomson, and John Wickerson. 2015. The Design and Implementation of a Verification Technique for GPU Kernels. ACM Trans. Program. Lang. Syst. 37, 3, Article 10 (May 2015), 49 pages. https://doi.org/10.1145/2743017

[7] François Bobot, Jean-Christophe Filliâtre, Claude Marché, and Andrei Paskevich. 2011. Why3: Shepherd Your Herd of Provers. In Boogie 2011: First International Workshop on Intermediate Verification Languages. Wrocław, Poland, 53-64. http://proval. lri.fr/publications/boogie11final.pdf

[8] Sylvie Boldo, François Clément, Jean-Christophe Filliítre, Micaela Mayero, Guillaume Melquiond, and Pierre Weis. 2014. Trusting Computations: A Mechanized Proof from Partial Differential Equations to Actual Program. Comput. Math. Appl. 68, 3 (Aug. 2014), 325-352. https://doi.org/10.1016/j.camwa.2014.06.004

[9] Jacob Burnim, Tayfun Elmas, George Necula, and Koushik Sen. 2011. NDSeq: Runtime Checking for Nondeterministic Sequential Specifications of Parallel Correctness. In Proceedings of the 32Nd ACM SIGPLAN Conference on Programming Language Design and Implementation (PLDI '11). ACM, New York, NY, USA, 401-414. https://doi.org/10.1145/1993498.1993545

[10] Cristian Cadar, Daniel Dunbar, and Dawson Engler. 2008. KLEE: Unassisted and Automatic Generation of High-coverage Tests for Complex Systems Programs. In Proceedings of the 8th USENIX Conference on Operating Systems Design and Implementation (OSDI'08). USENIX Association, Berkeley, CA, USA, 209-224. http://dl.acm.org/citation.cfm?id=1855741.1855756

[11] Pascal Cuoq, Florent Kirchner, Nikolai Kosmatov, Virgile Prevosto, Julien Signoles, and Boris Yakobowski. 2012. Frama-c. In Software Engineering and Formal Methods. Springer, Berlin, Heidelberg, 233-247.

[12] Steven J. Deitz, Bradford L. Chamberlain, and Lawrence Snyder. 2001. Eliminating Redundancies in Sum-of-product Array Computations. In Proceedings of the 15th International Conference on Supercomputing (ICS '01). ACM, New York, NY, USA, 65-77. https://doi.org/10.1145/377792.377807

[13] Azadeh Farzan, Andreas Holzer, Niloofar Razavi, and Helmut Veith. 2013. Con2Colic Testing. In Proceedings of the 2013 9th Joint Meeting on Foundations of Software Engineering (ESEC/FSE 2013). ACM, New York, NY, USA, 37-47. https: //doi.org/10.1145/2491411.2491453

[14] Patrice Godefroid. 1996. Partial-Order Methods for the Verification of Concurrent Systems - An Approach to the StateExplosion Problem. Lecture Notes in Computer Science, Vol. 1032. Springer.

[15] G. Gopalakrishnan, P. D. Hovland, C. Iancu, S. Krishnamoorthy, I. Laguna, R. A. Lethin, K. Sen, S. F. Siegel, and A. SolarLezama. 2017. Report of the HPC Correctness Summit, Jan 25-26, 2017, Washington, DC. ArXiv e-prints (May 2017). arXiv:cs.DC/1705.07478

[16] Ganesh Gopalakrishnan, Robert M. Kirby, Stephen Siegel, Rajeev Thakur, William Gropp, Ewing Lusk, Bronis R. De Supinski, Martin Schulz, and Greg Bronevetsky. 2011. Formal analysis of MPI-based parallel programs. Commun. ACM 54, 12 (Dec. 2011), 82-91. https://doi.org/10.1145/2043174.2043194 
[17] K.A. Hawick and D.P. Playne. 2010. Automated and parallel code generation for finite-differencing stencils with arbitrary data types. Procedia Computer Science 1, 1 (2010), 1795 - 1803. https://doi.org/10.1016/j.procs.2010.04.201 ICCS 2010.

[18] Daniel Jackson. 2006. Software Abstractions: Logic, Language, and Analysis. The MIT Press.

19] Shoaib Kamil, Alvin Cheung, Shachar Itzhaky, and Armando Solar-Lezama. 2016. Verified Lifting of Stencil Computations. SIGPLAN Not. 51, 6 (June 2016), 711-726. https://doi.org/10. $1145 / 2980983.2908117$

[20] Andreas Klöckner, Nicolas Pinto, Yunsup Lee, Bryan Catanzaro, Paul Ivanov, and Ahmed Fasih. 2011. PyCUDA and PyOpenCL: A Scripting-Based Approach to GPU Run-Time Code Generation. Parallel Comput. (2011).

[21] Navjot Kukreja, Mathias Louboutin, Felippe Vieira, Fabio Luporini, Michael Lange, and Gerard Gorman. 2016. Devito: automated fast finite difference computation. CoRR abs/1608.08658 (2016). http://arxiv.org/abs/1608.08658

[22] Monica D Lam, Edward E Rothberg, and Michael E Wolf. 1991. The cache performance and optimizations of blocked algorithms. In ACM SIGARCH Computer Architecture News, Vol. 19. ACM 63-74.

[23] Guodong Li, Peng Li, Geof Sawaya, Ganesh Gopalakrishnan, Indradeep Ghosh, and Sreeranga P. Rajan. 2012. GKLEE: Concolic Verification and Test Generation for GPUs. In Proceedings of the 17th ACM SIGPLAN Symposium on Principles and Practice of Parallel Programming (PPoPP '12). ACM, New York, 215-224. http://www.cs.utah.edu/fv/GKLEE.

24] Anders Logg, Kent-Andre Mardal, and Garth Wells. 2012. Automated Solution of Differential Equations by the Finite Element Method: The FEniCS Book. Springer Publishing Company, Incorporated.

[25] Anders Logg, Kent-Andre Mardal, and Garth Wells. 2012. Automated Solution of Differential Equations by the Finite Element Method: The FEniCS Book. Springer Publishing Company, Incorporated.

[26] Fabio Luporini, Ana Lucia Varbanescu, Florian Rathgeber, Gheorghe-Teodor Bercea, J. Ramanujam, David A. Ham, and Paul H. J. Kelly. 2015. Cross-Loop Optimization of Arithmetic Intensity for Finite Element Local Assembly. ACM Trans. Archit. Code Optim. 11, 4, Article 57 (Jan. 2015), 25 pages. https://doi.org/10.1145/2687415

[27] Message-Passing Interface Forum. 2015. MPI: A Message-Passing Interface Standard, Version 3.1. http://www.mpi-forum.org/docs/ docs.html. (4 June 2015).

[28] Aaron Meurer, Christopher P. Smith, Mateusz Paprocki, Ondřej Črtík, Sergey B. Kirpichev, Matthew Rocklin, AMiT Kumar, Sergiu Ivanov, Jason K. Moore, Sartaj Singh, Thilina Rathnayake, Sean Vig, Brian E. Granger, Richard P. Muller, Francesco Bonazzi, Harsh Gupta, Shivam Vats, Fredrik Johansson, Fabian Pedregosa, Matthew J. Curry, Andy R. Terrel, Štěpán Roučka, Ashutosh Saboo, Isuru Fernando, Sumith Kulal, Robert Cimrman, and Anthony Scopatz. 2017. SymPy: symbolic computing in Python. PeerJ Computer Science 3 (Jan. 2017), e103. https://doi.org/ 10.7717 /peerj-cs.103

29] Michael Lange, Navjot Kukreja, Fabio Luporini, Mathias Louboutin, Charles Yount, Jan Hückelheim, and Gerard J. Gorman. 2017. Optimised finite difference computation from symbolic equations. In Proceedings of the 15th Python in Science Conference, Katy Huff, David Lippa, Dillon Niederhut, and M Pacer (Eds.). $89-96$.

[30] Takayuki Muranushi, Hideyuki Hotta, Junichiro Makino, Seiya Nishizawa, Hirofumi Tomita, Keigo Nitadori, Masaki Iwasawa, Natsuki Hosono, Yutaka Maruyama, Hikaru Inoue, Hisashi Yashiro, and Yoshifumi Nakamura. 2016. Simulations of Belowground Dynamics of Fungi: 1.184 Pflops Attained by Automated Generation and Autotuning of Temporal Blocking Codes. In Proceedings of the International Conference for High Performance Computing, Networking, Storage and Analysis (SC '16). IEEE Press, Piscataway, NJ, USA, Article 3, 11 pages. http://dl.acm.org/citation.cfm?id=3014904.3014908

[31] John Nickolls, Ian Buck, Michael Garland, and Kevin Skadron. 2008. Scalable Parallel Programming with CUDA. Queue 6, 2 (March 2008), 40-53. https://doi.org/10.1145/1365490.1365500

[32] OpenMP Architecture Review Board. 2011. OpenMP Application Program Interface, Version 3.1. http://www.openmp.org/ mp-documents/OpenMP3.1.pdf. (July 2011).
[33] Corina S. Pasareanu and Neha Rungta. 2010. Symbolic PathFinder: symbolic execution of Java bytecode. In Proceedings of ASE. ACM, New York, NY, USA, 179-180.

[34] Zvonimir Rakamarić and Michael Emmi. 2014. SMACK: Decoupling Source Language Details from Verifier Implementations. In Computer Aided Verification - 26th International Conference, CAV 2014, Held as Part of the Vienna Summer of Logic, VSL 2014, Vienna, Austria, July 18-22, 2014. Proceedings (Lecture Notes in Computer Science), Armin Biere and Roderick Bloem (Eds.), Vol. 8559. Springer, 106-113.

[35] Florian Rathgeber, David A. Ham, Lawrence Mitchell, Michael Lange, Fabio Luporini, Andrew T. T. Mcrae, Gheorghe-Teodor Bercea, Graham R. Markall, and Paul H. J. Kelly. 2016. Firedrake: Automating the Finite Element Method by Composing Abstractions. ACM Trans. Math. Softw. 43, 3, Article 24 (Dec. 2016), 27 pages. https://doi.org/10.1145/2998441

[36] Florian Rathgeber, David A. Ham, Lawrence Mitchell, Michael Lange, Fabio Luporini, Andrew T. T. Mcrae, Gheorghe-Teodor Bercea, Graham R. Markall, and Paul H. J. Kelly. 2016. Firedrake: Automating the Finite Element Method by Composing Abstractions. ACM Trans. Math. Softw. 43, 3, Article 24 (Dec. 2016), 27 pages. https://doi.org/10.1145/2998441

[37] Patrick J Roache. 2002. Code verification by the method of manufactured solutions. Transactions-American Society of $\mathrm{Me}$ chanical Engineers Journal of Fluids Engineering 124, 1 (2002), $4-10$.

[38] Markus Schordan, Pei-Hung Lin, Dan Quinlan, and Louis-Noël Pouchet. 2014. Verification of Polyhedral Optimizations with Constant Loop Bounds in Finite State Space Computations. Springer Berlin Heidelberg, Berlin, Heidelberg, 493-508. https: //doi.org/10.1007/978-3-662-45231-8_41

[39] Koushik Sen. 2007. Concolic Testing. In Proceedings of the Twenty-second IEEE/ACM International Conference on Automated Software Engineering (ASE 'O7). ACM, New York, NY, USA, 571-572. https://doi.org/10.1145/1321631.1321746

[40] Stephen F. Siegel, Manchun Zheng, Ziqing Luo, Timothy K. Zirkel, Andre V. Marianiello, John G. Edenhofner, Matthew B. Dwyer, and Michael S. Rogers. 2015. CIVL: The Concurrency Intermediate Verification Language. In Proceedings of the International Conference for High Performance Computing, Networking, Storage and Analysis (SC '15). ACM, New York, Article 61, 12 pages. https://doi.org/10.1145/2807591.2807635

[41] Peter Vincent, Freddie Witherden, Brian Vermeire, Jin Seok Park, and Arvind Iyer. 2016. Towards Green Aviation with Python at Petascale. In Proceedings of the International Conference for High Performance Computing, Networking, Storage and Analysis (SC '16). IEEE Press, Piscataway, NJ, USA, Article 1, 11 pages. http://dl.acm.org/citation.cfm?id=3014904.3014906

The submitted manuscript has been created by UChicago Argonne, LLC, Operator of Argonne National Laboratory ("Argonne"). Argonne, a U.S. Department of Energy Office of Science laboratory, is operated under Contract No. DE-AC02-06CH11357. The U.S. Government retains for itself, and others acting on its behalf, a paid-up nonexclusive, irrevocable worldwide license in said article to reproduce, prepare derivative works, distribute copies to the public, and perform publicly and display publicly, by or on behalf of the Government. The Department of Energy will provide public access to these results of federally sponsored research in accordance with the DOE Public Access Plan http://energy.gov/downloads/ doe-public-access-plan. 